filgotinib also reduced the B- and T-cell development cytokine IL-7. In contrast, IL-8 was not affected by filgotinib. Reductions in MIP $1 \alpha$, MIP $1 \beta$ and GM-CSF are in line with a down modulation of innate immune activity.

Conclusions: Treatment of RA patients with filgotinib monotherapy resulted in significant reduction in the levels of a broad range of cytokines related to $T_{H} 1$, $T_{H} 2, T_{H} 17$ and potentially $B$ cells, as well as innate immunity. This observed anti-inflammatory activity of filgotinib is consistent with its efficacy in RA patients. References:

[1] Kavanaugh A et al. Ann Rheum Dis 2016;0:1-11.doi:10.1136/annrheumdis2016-210105.

Disclosure of Interest: A. Kavanaugh Consultant for: Galapagos, Pfizer, Abbvie, Amgen, Celgene, Janssen, Novartis, Eli Lilly, UCB, A. Van der Aa Employee of: Galapagos NV, C. Jamoul Employee of: Galapagos NV, W. Li Employee of: Gilead Sciences, L. Goyal Employee of: Gilead Sciences, Y. Pan Employee of: Gilead Sciences, P. Harrison Employee of: Galapagos NV, C. Tasset Employee of: Galapagos NV, J. Tarrant Employee of: Gilead Sciences, R. Galien Employee of: Galapagos SASU

DOI: 10.1136/annrheumdis-2017-eular.5814

\section{THU0183 IMPROVED ADHERENCE TO NEWLY PRESCRIBED DMARDS WITH CO-PRESCRIPTION WITH LOW DOSE STEROIDS IN RHEUMATOID ARTHRITIS PATIENTS ATTENDING CLINIC AT A DISTRICT GENERAL HOSPITAL IN THE UK}

A. Nugaliyadde ${ }^{1,2}, \mathrm{~K}$. Culfear ${ }^{2}, \mathrm{~A}$. Nandagudi ${ }^{2}, \mathrm{~A}$. Bharadwaj ${ }^{2} .{ }^{1}$ Department of Rheumatology, Teaching Hospital Kandy, Kandy, Sri Lanka; ${ }^{2}$ Department of Rheumatology, Basildon and Thurrock University Hospital, Basildon, United Kingdom

Background: Non-adherence to DMARDs is associated with disease flares and increased disability. Adherence rates to prescribed medicine regimes in people with Rheumatoid Arthritis vary from $30-80 \%$ in different studies. Improving adherence to therapy leads to better disease outcome and reduced costs associated with management of RA.

Objectives: This study was carried out as a pilot study to look at the effect of co-prescription of steroids on the adherence and side effects to newly prescribed DMARDS in patients with rheumatoid arthritis.

Methods: This is a prospective, observational cohort study, for the duration of three months per participant. Patients were selected sequentially from those attending outpatient clinic at Basildon Hospitals with a confirmed diagnosis of rheumatoid arthritis (ACR/EULAR criteria), and had been planned to start on a new DMARD by the treating physician. Baseline data included demographics, disease characteristics and data regarding steroid co- prescription including route, dose and duration. Patients were reviewed at three months to look at DMARD adherence defined by continuation of the DMARD. We looked at the side effect profile as possible contributing factor to non-adherence. The effect of co-prescription with steroids and other demographic data on treatment duration was investigated using Kaplan-Meier survival plots. Logistic regression analysis was used to investigate the effect of co- prescription of steroids on continuation of medication.

Results: Fifty one patients were recruited to the study. Median age at the time of enrolment was 61 years (IQR 46-71), 73\% were females and 92\% were caucasians. seventy percent of the patients were seropositive and DMARD naïve with a mean DAS CRP at recruitment of $4.13(1.21)$. Seventeen (33\%) patients were co-prescribed with steroids at the initiation of DMARDs. Out of these $59 \%$ $(n=10)$ were DMARD naïve. Thirteen patients received a tapering dose of oral prednisolone with a mean starting dose of $13.8 \mathrm{mg}$ daily (range $3 \mathrm{mg}-20 \mathrm{mg}$ ) for a mean duration of 10.8 weeks. Two patients received oral prednisolone $5 \mathrm{mg}$ daily for 12 week. The mean cumulative dose of oral prednisolone prescribed was $632.2 \mathrm{mg}$. The two remaining patients received $120 \mathrm{mg}$ of depomedrone IM. The non adherence rate for our cohort was 35\%,6\% for patients co-prescribed with steroids versus $50 \%$ for patients who were not co-prescribed with steroids. The odds ratio for likelihood of discontinuation for patients who were not co-prescribed with steroids versus patients who were, was $16(1.94-134.5, \mathrm{p}=0.011)$.

At the end of three months $25 \%$ of the whole cohort, $12 \%$ of the patients who were co-prescribed with steroids versus $32 \%$ of the patients who were not co-prescribed with steroids reported side effects to the DMARD initiated. the odds ratio for reporting side effects with steroid co-prescription was 0.28 (0.054-1.438, $\mathrm{p}=0.12$ ).

Conclusions: Co-prescription of low dose steroids with initiation of DMARDs increases the chances of adherence and possibly reduces the side-effect profile References:

[1] van den Bemt BJ, Zwikker HE, van den Ende CH. Medication adherence in patients with rheumatoid arthritis: a critical appraisal of the existing literature. Expert Rev Clin Immunol. 2012;8(4):337-351.

Disclosure of Interest: None declared

DOI: 10.1136/annrheumdis-2017-eular.2182

\section{THU0184 ADHERENCE PROFILES TO METHOTREXATE OF PATIENTS WITH RHEUMATOID ARTHRITIS (RA) ELIGIBLE FOR BIOLOGICS: TYPOLOGIES FROM FORGET, A CROSS-SECTIONAL SURVEY OF 244 PATIENTS}

C. Beauvais ${ }^{1}$, V. Hautin-Monteil ${ }^{2}$, M.-C. Ducrot ${ }^{3}$, R.-M. Flipo ${ }^{4}{ }^{1}$ Rheumatology Department, Saint Antoine Hospital, APHP, Paris; ${ }^{2}$ Roche France; ${ }^{3}$ Agence A+A, Boulogne-Billancourt; ${ }^{4}$ Rheumatology department, Roger Salengro Hospital, Lille, France

Background: Adherence to Methotrexate (MTX) is not optimal in RA patients [1]. Conflicting determinants of adherence have been identified in literature. Our hypothesis was that the discordant results were related to different typologies of patients.

Objectives: Determine the Methotrexate adherence profiles of patients with RA eligible for biologics.

Methods: The FORGET survey carried out in 2016 was aimed to assess the MTX adherence rate of RA patients, insufficient responders to MTX, biologic-naive, when an initiation of biologics was being considered. Non-adherence was defined as a compliance rate $<80 \%$ according to the CQR 19 (Compliance Questionnaire for Rheumatology) [2]. The factors tested were socio-demographic characteristics, DAS 28, RAID, CQR responses, beliefs, voluntary or involuntary dose skipping, social and medical support.

Results: Of the 244 patients analyzed, the non-adherence rate was $34 \%$. The rather weak correlation between adherence (CQR) and the disease impact (RAID) tended to confirm the hypothesis of different profiles. Four typologies of patients were determined. Groups G1 and G2 were non-adherent patients with high (G1) or lower (G2) impact. Groups G3 and G4 were patients with good adherence with high (G3) or lower impact (G4. Significant adherence factors were found for these 4 groups $(p<0.01)$ (table)

\begin{tabular}{|c|c|c|c|c|}
\hline & \multicolumn{2}{|c|}{$\begin{array}{l}\text { Non-adherent } \\
\text { patients }\end{array}$} & \multicolumn{2}{|c|}{$\begin{array}{l}\text { Adherent } \\
\text { patients }\end{array}$} \\
\hline & G1 & $\mathrm{G} 2$ & G3 & G4 \\
\hline$\%$ & 14 & 19 & 33 & 34 \\
\hline CQR 19 (\%) & 21 & 40 & 78 & 88 \\
\hline RAID (0-10) mean & 6,7 & 4,6 & 6,3 & 3,7 \\
\hline Skipped doses (\%) & 66 & 55 & 15 & 11 \\
\hline Skipped does without doctor's recommendation (\%) & 38 & 41 & 3 & 3 \\
\hline I comorbidity or more (\%) & 62 & 68 & 57 & 45 \\
\hline Depression (\%) & 31 & 14 & 12 & 3 \\
\hline Anxiety & 16 & 9 & 9 & 1 \\
\hline Good information received (\%) & 69 & 83 & 81 & 93 \\
\hline I find constrains to take may treatment (\% yes) & 59 & 61 & 21 & 6 \\
\hline I tolerate my treatment badly (\% yes) & 47 & 18 & 16 & 18 \\
\hline I think every day about may arthritis (\% yes) & 83 & 63 & 88 & 53 \\
\hline \multicolumn{5}{|l|}{ I find it hard to keep may professional obligations } \\
\hline because of my arthritis (\% yes) & 79 & 38 & 65 & 32 \\
\hline I feel well informed about my arthritis (\% yes) & 69 & 83 & 81 & 93 \\
\hline I had support from my relatives (\% yes) & 55 & 55 & 82 & 96 \\
\hline \multicolumn{4}{|l|}{ My treatment is doing me more harm than good (\% no } & 71 \\
\hline \multicolumn{5}{|l|}{ The most important reason to take my treatment is that } \\
\hline I can still do what I want to do (\% complete agreement) & 6,3 & 11,4 & 17,2 & 60,5 \\
\hline \multicolumn{5}{|l|}{ I do not expect miracles from my treatment (\% no } \\
\hline agrement at all) & 0 & 0 & 10,3 & 23 \\
\hline
\end{tabular}

Conclusions: Four adherence profiles to Methotrexate have been identified. Among the non-adherent patients, 2 topologies are opposed: 1 - patients in state of suffering, with low support from relatives, negative beliefs and significant professional impact. 2-patients with less disease impact, who perceived their treatment with constraints although well tolerated. Detection of patients' profiles may allow targeted strategies to improve or maintain adherence.

References:

[1] DiBenedetti D Rheumatol Ther 2015.

[2] de Klerk E et al, J Rheumatol 1999.

Acknowledgements: This study was funded by Chugai Pharma France. Disclosure of Interest: C. Beauvais: None declared, V. Hautin-Monteil Employee of: Roche France, M.-C. Ducrot: None declared, R.-M. Flipo: None declared DOI: 10.1136/annrheumdis-2017-eular.5523

\section{THU0185 COMPARISON OF TOFACITINIB SAFETY AND EFFICACY IN RHEUMATOID ARTHRITIS PATIENTS WITH INADEQUATE RESPONSE TO CONVENTIONAL SYNTHETIC DMARDS, OR TO ONE OR MORE BIOLOGICAL DMARDS}

C. Charles-Schoeman ${ }^{1}$, J. Kremer ${ }^{2}$, S. Krishnaswami ${ }^{3}$, K. Soma ${ }^{4}$, J. Geier ${ }^{4}$, R. Zhang ${ }^{4}$, S. Strengholt ${ }^{4}$, G. Burmester ${ }^{5} .{ }^{1}$ University of California, LoS Angeles, CA; ${ }^{2}$ Albany Medical College and the Center for Rheumatology, Albany, NY; ${ }^{3}$ Pfizer Inc, Groton, CT; ${ }^{4}$ Pfizer Inc, New York, NY, United States; ${ }^{5}$ Charité University Medicine Berlin, Berlin, Germany

Background: Tofacitinib is an oral JAK inhibitor for the treatment ( $\mathrm{tx}$ ) of rheumatoid arthritis (RA). Studies have shown diminishing response to tx in RA patients (pts) when cycling through TNF inhibitors. Prior analyses assessed tofacitinib in csDMARD-inadequate response (IR) pts vs overall bDMARD-IR pts. 
Objectives: To compare tofacitinib safety and efficacy in RA pts who have previously failed tx (lack of efficacy and/or safety reasons) with csDMARDs, with pts who failed tx with either 1 or $\geq 2$ prior bDMARDs.

Methods: Data from pts who received $\geq 1$ dose of tofacitinib in 19 RA studies up to 96 months (2 Phase [P] 1; 9 P2; 6 P3; 2 LTE studies [1 LTE ongoing; data as of March 2015]) were used in this analysis. Data were pooled across all 19 studies for safety assessments in the All RA population: csDMARD-IR, $n=4377$; bDMARD-IR, $n=838$ ( 1 bDMARD-IR, $n=533$; $\geq 2$ bDMARD-IR $n=305$ ). Safety was also assessed up to 24 months in pts randomised to tofacitinib 5 or $10 \mathrm{mg} \mathrm{BID}$ or placebo $(\mathrm{PBO})$ in a pooled $\mathrm{P} 2 / \mathrm{P} 3$ randomised controlled trial $(\mathrm{RCT})$ population (8 P2, 6 P3 studies; csDMARD-IR, $n=3328$; bDMARD-IR, $n=782$ ). Incidence rates (pts with events/100 pt-years) were calculated for serious AEs (SAEs), serious infections (SIs) and herpes zoster (HZ). Efficacy was assessed by pts achieving ACR20 response and DAS28-4(ESR) $\leq 3.2$ at Month 3 in a pooled P3 RCT population (csDMARD-IR, $n=2375$; bDMARD-IR, $n=664$ ).

Results: Prior to tofacitinib tx, bDMARD-IR pts had longer RA duration, greater disease burden and more corticosteroid use vs csDMARD-IR pts. SAEs were more common among bDMARD-IR vs csDMARD-IR pts in both the P2/P3 RCT and the All RA populations; SAE rates were not higher in pts failing $\geq 2$ bDMARDs vs 1 bDMARD (Table). Incidence rates for SIs were generally greater in pts with IR to bDMARDs vs csDMARDs in the All RA population, but generally lower in pts with IR to 1 or $\geq 2$ bDMARDs vs csDMARDs in the P2/P3 RCT population; incidence with $5 \mathrm{mg}$ BID was lower for $1 \mathrm{vs} \geq 2$ bDMARDs in the P2/P3 RCT population. Incidence rates for $\mathrm{HZ}$ were similar between pts with IR to csDMARDs or $1 \mathrm{bDMARD}$, but appeared numerically greater in pts with IR to $\geq 2$ bDMARDs in both the P2/P3 RCT and the All RA populations. A similar pattern was observed across tofacitinib and PBO groups. Efficacy at Month 3 in the P3 RCT population was greater with both tofacitinib doses vs PBO. Although absolute response was smaller in pts with IR to bDMARDs vs csDMARDs, generally similar efficacy was observed in pts with IR to 1 or $\geq 2$ bDMARDs (Table).

\begin{tabular}{|c|c|c|c|c|c|}
\hline \multicolumn{6}{|c|}{ Table. Selected safety and efficacy outcomes by prior failed treatment. } \\
\hline & & $\begin{array}{c}\text { cSDMARD-IR- } \\
\text { only }\end{array}$ & $\begin{array}{c}\text { Overall } \\
\text { bDMARD-IR }\end{array}$ & $\begin{array}{c}1 \\
\text { LDMARD-IR } \\
\end{array}$ & $\stackrel{\geq 2}{\geq 2}$ LDMARD-IRs \\
\hline \multicolumn{6}{|c|}{$\begin{array}{l}\text { P2/P3 RCT population (0-24 months): } \\
\text { incidence rate (pts with event/100 pt-years) (95\% Cl) }\end{array}$} \\
\hline \multirow{3}{*}{ SAES } & Tofacitinib $5 \mathrm{mg}$ BID & $12.4(10.3,14.8)$ & $12.6(7.8,19.3)$ & $12.7(6.8,21.7)$ & $12.6(5.4,24.7)$ \\
\hline & \begin{tabular}{|l} 
Tofacitimib $10 \mathrm{mg}$ BID \\
\end{tabular} & $9.7(7.9,11.8)$ & $12.5(7.6,19.3)$ & $12.1(6.3,21.1)$ & $13.1(5.6,25.7)$ \\
\hline & \begin{tabular}{|l|} 
Placebo \\
\end{tabular} & $11.5(7.6,16.8)$ & $20.5(9.8,37.7)$ & $19.8(7.3,43.1)$ & $21.6(5.9,55.4)$ \\
\hline \multirow{3}{*}{ SIs } & Tofacitinib $5 \mathrm{mg}$ BID & $3.4(24,4.7)$ & $1.7(0.4,5.0)$ & $0.9(0.0,5.2)$ & $2.9(0.4,10.6)$ \\
\hline & Tofacitinib $10 \mathrm{mg}$ BID & $3.4(24,4.7)$ & $3.0(1.0,7.1)$ & $3.0(0.6,8.7)$ & $2(0.4,11.4)$ \\
\hline & Placebo & $1.7(0.5,4.3)$ & $4.1(0.5,14.7)$ & $3.3(0.1,18.3)$ & $5.4(0.1,30.1)$ \\
\hline \multirow{3}{*}{$\mathrm{Hz}$} & Tofacitinib 5 mg BID & $3.9(28,5.3)$ & $5.3(2.4,10.0)$ & $2.8(0.6,8.3)$ & $9.1(3.4,19.9)$ \\
\hline & \begin{tabular}{|l|} 
Tofacitmib $10 \mathrm{mg} \mathrm{BID}$ \\
\end{tabular} & & $6.3(3$. & $6.2(2.3,13.4)$ & $6(1.8,16.8)$ \\
\hline & \begin{tabular}{|l|l|} 
Placebo \\
\end{tabular} & $2.6(09,5.5)$ & $0.0(0,0,7.5)$ & $0.0(0.0,12.1)$ & $0.0(0.0,19.8)$ \\
\hline \multicolumn{6}{|c|}{$\begin{array}{l}\text { All RA population (up to } 96 \text { months); } \\
\text { incidence rate (pts with event/100 pt-years) }(95 \% \text { Cl) }\end{array}$} \\
\hline \multirow{3}{*}{ SAEs } & Tofacitimib 5 mg BID & $9.7(8.9,10.6)$ & $14.8(11.4,189)$ & $15.9(11.5,21.3)$ & $12.8(7.7,20.0)$ \\
\hline & \begin{tabular}{|l|} 
Tofacitimib $10 \mathrm{mg} \mathrm{BID}$ \\
\end{tabular} & $9.7(9.0,10.4)$ & $11.6(10.0,13.3)$ & $11.8(9.8,14.0)$ & $11.3(8.8,14.3)$ \\
\hline & \begin{tabular}{|l|l|l} 
All tofacitit \\
\end{tabular} & & 12.2 & 12.6 & $3,14.3)$ \\
\hline \multirow{3}{*}{ sis } & Tofacitinib $5 \mathrm{mg}$ BID & $2.9(25,3,4)$ & $4.5(2.8,6.8)$ & $4.7(2.7,7.8)$ & $4.1(1.6,8.4)$ \\
\hline & \begin{tabular}{|l} 
Tofacitinib $10 \mathrm{mg}$ BID \\
\end{tabular} & $27(2,3,3.1)$ & $3.5(27,4,4)$ & $4.0(2.9,5.3)$ & $2.6(1.5,4.1)$ \\
\hline & \begin{tabular}{|l|l|l} 
All tofacitinib doses \\
\end{tabular} & $2.8(25,3.1)$ & $3.7(29,45)$ & $4.1(3.2,5.3)$ & $2.9(1.9,4.2)$ \\
\hline \multirow{3}{*}{$\mathrm{HZ}$} & Tofacitinib $5 \mathrm{mg} \mathrm{BID}$ & $3.7(3.2,4.3)$ & & 3.601 & $7.1(3.5,12.7)$ \\
\hline & \begin{tabular}{|l|l|} 
Tofacitinib $10 \mathrm{mg}$ BID \\
\end{tabular} & $4.2(3.8,4.7)$ & $4.4(35,55)$ & $4.1(3.0,5.5)$ & $4.9(3 ., 7.0)$ \\
\hline & \begin{tabular}{|l|} 
All tofacitinib doses \\
\end{tabular} & $4.0(3.7,4.4)$ & $4.5(3.6,5.5)$ & $4.0(3.0,5.2)$ & $5.4(39,7.2)$ \\
\hline \multicolumn{6}{|c|}{ Efficacy endpoints in the P3 RCT population at Month $3 ; \%$ of pts } \\
\hline \multirow{3}{*}{ ACR20 } & \begin{tabular}{|l} 
Tofacitinib $5 \mathrm{mg}$ BID \\
\end{tabular} & $59.4^{* * *+}$ & $43.5 \%$ & $45.3^{*}$ & $40.6^{\circ}$ \\
\hline & \begin{tabular}{|l|l|} 
Tofacitinib $10 \mathrm{mg}$ BID \\
\end{tabular} & $65.4^{* * * *}$ & $50.6^{6 * 4}$ & $50.6^{* * * *}$ & $50.6^{\text {stot }}$ \\
\hline & \begin{tabular}{|l|l|l|l} 
Placebo \\
\end{tabular} & 27.1 & 24.6 & 27.2 & 20.0 \\
\hline \multirow{3}{*}{$\begin{array}{l}\text { DAS28- } \\
\text { t(ESR) } \\
3.2 .2\end{array}$} & \begin{tabular}{|l} 
Tofacitinib $5 \mathrm{mg}$ BID \\
\end{tabular} & $15.9 \%$ & $12.7^{*}$ & 11.9 & $14.0^{*}$ \\
\hline & Tofacitinib $10 \mathrm{mg}$ BID & $21.1 \%$ & $15.8^{* *}$ & $15.7^{\circ}$ & $16.5^{*}$ \\
\hline & Placebo & 3.9 & 4.9 & 5.8 & 3.4 \\
\hline
\end{tabular}

Conclusions: SAEs and SIEs were more common in tofacitinib- and PBO-treated pts with IR to bDMARDs vs csDMARDs; increased risk was generally not observed with increasing number of prior bDMARDs. HZ risk appeared greater with $\geq 2$ bDMARDs vs 1 bDMARD. Efficacy was greater with tofacitinib vs PBO for csDMARD-IR and bDMARD-IR pts, with similar response observed in pts with IR to $\geq 2$ or 1 bDMARD. These data support the use of tofacitinib in different lines of therapy, although the analysis is limited by smaller sample size in some groups.

Acknowledgements: This study was sponsored by Pfizer Inc. Editorial support was provided by N Jones of CMC and was funded by Pfizer Inc.

Disclosure of Interest: C. Charles-Schoeman Grant/research support from: AbbVie, Bristol-Myers Squibb, Pfizer Inc, Consultant for: Amgen, Pfizer Inc, Regeneron-Sanofi, J. Kremer Shareholder of: Corrona, LLC, Consultant for: AbbVie, Amgen, Bristol-Myers Squibb, Genentech, Eli Lilly, Pfizer Inc, Employee of: Corrona, LLC, S. Krishnaswami Shareholder of: Pfizer Inc, Employee of: Pfizer Inc, K. Soma Shareholder of: Pfizer Inc, Employee of: Pfizer Inc, J. Geier Shareholder of: Pfizer Inc, Employee of: Pfizer Inc, R. Zhang Shareholder of: Pfizer Inc, Employee of: Pfizer Inc, S. Strengholt Shareholder of: Pfizer Inc, Employee of: Pfizer Inc, G. Burmester Grant/research support from: UCB, Consultant for: AbbVie, Bristol-Myers Squibb, Eli Lilly, Hexal, Janssen, Medimmune, MSD,
Novartis, Pfizer Inc, Roche, Sanofi, Speakers bureau: AbbVie, Bristol-Myers Squibb, Hexal, MSD, Novartis, Pfizer Inc, Roche

DOI: 10.1136/annrheumdis-2017-eular.2346

\section{THU0186 MAGNITUDE AND DURATION OF EARLY RESPONSE WITH TOFACITINIB: POST-HOC ANALYSIS OF TWO PHASE 3 , PLACEBO-CONTROLLED STUDIES}

D. Aletaha ${ }^{1}$, A. Kivitz ${ }^{2}$, G. Valenzuela ${ }^{3}$, J. Tesser $^{4}$, S. Hays ${ }^{5}$, H. Li $^{5}$, C.A. Connell ${ }^{6}$, E. Bananis ${ }^{5}$, A. Soonasra ${ }^{5}$, J.S. Smolen ${ }^{7} .{ }^{1}$ Department of Internal Medicine 3, Medical University of Vienna, Vienna, Austria; ${ }^{2}$ Altoona Center for Clinical Research, Duncansville, PA; ${ }^{3}$ Integral Rheumatology \& Immunology Specialists, Fort Lauderdale, FL; ${ }^{4}$ Arizona Arthritis \& Rheumatology Associates, Glendale, AZ; ${ }^{5}$ Pfizer Inc, Collegeville, PA; ${ }^{6}$ Pfizer Inc, Groton, CT, United States; ${ }^{7}$ Division of Rheumatology, Medical University of Vienna and Hietzing Hospital, Vienna, Austria

Background: Tofacitinib is an oral Janus kinase inhibitor for the treatment of RA. ORAL Solo (NCT00814307) and ORAL Sync (NCT00856544) were two Phase 3 index studies that demonstrated the efficacy of tofacitinib in adult patients (pts) with RA who were DMARD inadequate responders (DMARD-IR). Early onset of effect is a clinically meaningful endpoint.

Objectives: This post hoc analysis examined the magnitude and durability of early response to tofacitinib in ORAL Solo and ORAL Sync.

Methods: ORAL Solo and ORAL Sync were double-blind, placebo (PBO)controlled, parallel-group studies in pts with active RA and an inadequate response to $\geq 1$ conventional synthetic (cs) or biologic (b) DMARDs. Pts were randomised to tofacitinib $5 \mathrm{mg}$ BID, tofacitinib $10 \mathrm{mg}$ BID, PBO advanced to tofacitinib $5 \mathrm{mg}$ BID, or PBO advanced to tofacitinib $10 \mathrm{mg}$ BID, either as monotherapy in ORAL Solo or with background csDMARDs in ORAL Sync. In ORAL Solo, pts randomised to PBO were advanced to tofacitinib at Month (M) 3; in ORAL Sync, pts randomised to PBO were advanced to tofacitinib at M3 (nonresponders) or M6 (all other pts). In this post hoc analysis, the following clinical efficacy data for pts on tofacitinib or PBO (prior to advancement to tofacitinib) \pm csDMARDs, were evaluated at Week 2, M3 and M6 (ORAL Sync only; no M6 PBO comparison in ORAL Solo): change from baseline (CFB) in Clinical Disease Activity Index (CDAI) $>12,{ }^{1} \mathrm{HAQ}-\mathrm{DI} \mathrm{CFB} \geq 0.22, \mathrm{CDAl} \geq 50 \%$ improvement from baseline, $\mathrm{CDAl}>70 \%$ improvement from baseline, $\mathrm{CDAl}>85 \%$ improvement from baseline, Functional Assessment of Chronic Illness Therapy-Fatigue (FACIT-F) score improvement from baseline $\geq 4$, Pain visual analogue scale (VAS) score $\mathrm{CFB}>10$. This analysis was post hoc and multiplicity adjustment was done.

Results: Clinical efficacy endpoint data are summarised in the Table. At Week 2, more patients receiving tofacitinib 5 or $10 \mathrm{mg}$ BID \pm cSDMARDs (compared with $\mathrm{PBO}+$ csDMARDs) achieved a CDAI CFB $>12$, $\mathrm{HAQ}-\mathrm{DI} \mathrm{CFB} \geq 0.22, \mathrm{CDAl} \geq 50 \%$ improvement from baseline and pain VAS CFB $\geq 10$. By M3, more pts receiving tofacitinib 5 or $10 \mathrm{mg} \mathrm{BID} \pm$ csDMARDs (compared with PBO \pm csDMARDs) achieved the efficacy outcomes measured including improvements from baseline in FACIT-F scores $\geq 4, \mathrm{CDAl} \geq 50 \%, \mathrm{CDAl} \geq 70 \%$ and $\mathrm{CDAl} \geq 85 \%$. Responses attained at M3 were maintained or increased at M6.

\begin{tabular}{|c|c|c|c|c|c|c|c|}
\hline \multirow[b]{2}{*}{$\begin{array}{l}\text { Improvem } \\
(95 \% \text { CI) }\end{array}$} & \multirow[b]{2}{*}{ pe, \%pts } & \multicolumn{3}{|c|}{ ORAL Solo } & \multicolumn{3}{|c|}{ ORAL Syae } \\
\hline & & $\begin{array}{c}\text { Tofacitinib } \\
5 \text { ma } B D \\
(N=241)\end{array}$ & $\begin{array}{c}\text { Tofacitinib } \\
10 \mathrm{mg} B \mathrm{BD} \\
(\mathrm{N}=243)\end{array}$ & $\begin{array}{c}\text { PBO } \\
(N=122)\end{array}$ & $\begin{array}{c}\text { Tofacitinib } \\
5 \text { mg BID } \\
+ \text { caDMARDs } \\
\text { (N=312) }\end{array}$ & $\begin{array}{c}\text { Tofacitinib } \\
100 \text { mBD } \\
+ \text { caDMARDs } \\
\text { (N=315) }\end{array}$ & $\begin{array}{c}\stackrel{\text { PBO }}{+} \\
\text { CADMARDs } \\
\text { (N=158) }\end{array}$ \\
\hline \multirow{3}{*}{$\mathrm{CDAI}>12$} & Week 2 & $37.1(31.0,43.6)^{*}$ & $43.9(37.5,50.5)^{*}$ & $18.5(12.0,26.6)$ & $30.4(25.3,35.9)^{*}$ & $36.8(31.3,42.5)^{*}$ & $17.4(11.8,24.3)$ \\
\hline & Month 3 & $71.6(65.4,77.2)^{*}$ & $75.5(69.6,80.8)^{*}$ & $42.5(33.5,51.9)$ & $56.0\left(50.3,61 . \sigma^{*}\right.$ & $64.7(59.1,70.1)^{*}$ & $32.5(252 ., 40.4)$ \\
\hline & Month 6 & $79.5(74.4,84.6)$ & $78.8(33.7,84.0)$ & $\mathrm{NA}$ & $53.7(48.0,59.4)^{*}$ & $62.1(56.4,67.6)^{*}$ & $34.4(27.0,42.4)$ \\
\hline \multirow[t]{3}{*}{ HAQ-DI $\approx 0.22$} & Week 2 & $49.0(42.5,55.5)^{*}$ & $50.6(44.1,57.2)^{*}$ & $37.8(29.1,47.2)$ & $45.6(40.0,51.4)^{*}$ & $53.1(47.3,58.8)^{*}$ & $34.2(26.8,42.2)$ \\
\hline & Month 3 & $59.6(53.1,65.9)^{*}$ & $65.3(589,71.3)^{*}$ & $39.2(30.4,485)$ & $49.0(433,54.7)^{*}$ & $57.1(51.4,62.7)^{*}$ & $23.7(173,31.2)$ \\
\hline & Month 6 & $67.5(61.6,73.4)$ & $66.1(60.1,72.1)$ & $\mathrm{NA}$ & $49.7(440,55.4)^{*}$ & $55.5(49.8,61.2)^{*}$ & $23.7(173,31.2)$ \\
\hline \multirow[t]{3}{*}{$\mathrm{CDAI} \geq 50 \%$} & Week 2 & $19.8(15.0,25.5)^{*}$ & $25.1(19.7,31.1)^{*}$ & $8.4(4.1,14.9)$ & $18.0(13.8,22.7)^{*}$ & $20.5(16.1,25.5)^{*}$ & $9.0(5.0,14.7)$ \\
\hline & Month 3 & $57.3(50.8,63.7)^{*}$ & $58.1(51.6,64.4)^{*}$ & $25.8(183,34.6)$ & $45.3(39.7,51.0)^{*}$ & $56.5(50.8,62.2)^{*}$ & $22.3(16.1,29.6)$ \\
\hline & Mouth6 & $68.6(62.7,74.5)$ & $66.4(60.4,72.4)$ & $\mathrm{NA}$ & $48.5(42.9,54.3)^{*}$ & $58.8(53.1,64.4)^{*}$ & $29.3(22.3,37.1)$ \\
\hline \multirow[t]{3}{*}{$\mathrm{CDAI} \geq 70 \%$} & Week 2 & $5.1(2.6,8.7)$ & $11.3(.6,16.0)^{*}$ & $2.5(0.5,7.2)$ & $4.9(2.8,8.0)$ & $8.6(5.7,12.4)^{*}$ & $3.2(1.1,7.4)$ \\
\hline & Month 3 & $33.1(27.1,39.4)^{*}$ & $40.7(34,4,47.2)^{*}$ & $14.2(85,21.7)$ & $24.3(19.6,29.5)^{*}$ & $31.4(26.2,36.9)^{*}$ & $7.6(4.0,13.0)$ \\
\hline & Month 6 & $41.8(35.6,48.1)$ & $49.0(42.7,55.3)$ & $\mathrm{NA}$ & $31.4(263,369)^{*}$ & $379(325,43.6)^{x}$ & $16.6(11.1,23.3)$ \\
\hline \multirow[t]{3}{*}{$\mathrm{CDAI} \geq 85 \%$} & Week 2 & $2.1(0.7,4.9)$ & $5.0(2.6,8.6)$ & $2.5(0.5,7.2)$ & $1.0(0.2,2.8)$ & $3.3(1.6,6.0)^{*}$ & $0.7(0.0,3.5)$ \\
\hline & Month 3 & $14.6(10.4,19.8)^{*}$ & $17.8(132,23.3)^{*}$ & $5.8(2.4,11.7)$ & $9.4(6.4,13.2)^{*}$ & $11.4(8.1,15.6)^{*}$ & $1.3(0.2,45)$ \\
\hline & Month 6 & $22.2(16.9,27.4)$ & $27.0(21.4,32.6)$ & $\mathrm{NA}$ & $10.7(7.5,14.7)$ & $18.3(14.1,23.1)^{*}$ & $5.7(2.7,10.6)$ \\
\hline \multirow[t]{3}{*}{ FACIIF $: 4$} & Month 1 & $\mathrm{NA}$ & NA & $\mathrm{NA}$ & $46.2(40.4,52.0)$ & $58.1(52.3,63.7)^{\circ}$ & $39.0(31.2,47.1)$ \\
\hline & Month 3 & $61.2(54.7,67.4)^{*}$ & $63.4(56.8,69.6)^{*}$ & $42.2(33.1,51.8)$ & $38.6(33.1,44.3)^{*}$ & $51.2(45.4,56.9)^{x}$ & $21.8(15.6,29.1)$ \\
\hline & Mouth 6 & $59.2(53.0,65.5)$ & $62.4(56.2,68.6)$ & $\mathrm{NA}$ & $40.9\left(35.3,46.6^{*}\right.$ & $47.2(41.5,53.0)^{*}$ & $21.2(15.0,28.4)$ \\
\hline \multirow[t]{3}{*}{ Pain VAS $\geqq 10$} & Week 2 & $57.3(50.8,63.7)^{*}$ & $61.1(54.6,67.3)^{*}$ & $41.2(32.2,50.6)$ & $46.3(40.6,52.0)^{*}$ & $52.9(47.2,58.6)^{*}$ & $36.5(29.0,44.6)$ \\
\hline & Month 3 & $693(63.1,75.1)^{*}$ & $73.9(67.8,79.3)^{*}$ & $425(33.5,51.9)$ & $50.0(443,55.7)^{*}$ & $57.5(51.7,63.1)^{*}$ & $28.7(21.7,36.4)$ \\
\hline & Mouth 6 & $71.0(65.2,76.7)$ & $71.0(65.2,76.7)$ & $\mathrm{NA}$ & $48.4(42.7,54.1)^{*}$ & $56.2(50.4,61.8)^{*}$ & $30.6(23.5,38.4)$ \\
\hline
\end{tabular}

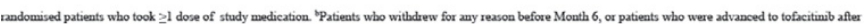
Mouth 3 (only for ORAL Syac) bud their values on or after withdrawing or advancing set to Non-Reepponse in this anlyyis. N= total number in each treatment proup aumber of patients available for the analysis may vary for individual endpoints. PBO group included only those patients who had not yet ad

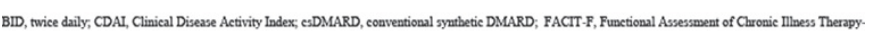

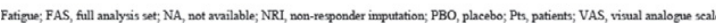

Conclusions: DMARD-IR pts with active RA receiving tofacitinib \pm csDMARDs appeared to show greater improvements compared with $\mathrm{PBO}$ in clinical disease 